\title{
Simultaneous Water Filling in Mutually Interfering Systems
}

\author{
Otilia Popescu, Dimitrie C. Popescu, and Christopher Rose
}

\begin{abstract}
In this paper we investigate properties of simultaneous water filling for a wireless system with two mutually interfering transmitters and receivers with non-cooperative coding strategies. This is slightly different from the traditional interference channel problem which assumes that transmitters cooperate in their respective coding strategies, and that interference cancellation can be performed at the receivers. In this non-cooperative setup, greedy capacity optimization by individual transmitters through various algorithms leads to simultaneous water filling fixed points where the spectrum of the transmit covariance matrix of one user water fills over the spectrum of its corresponding interference-plus-noise covariance matrix, and in our paper we study the properties of these fixed points. We show that at a simultaneous water filling point the eigenvectors of transmit covariance matrices at each receiver are aligned, and identify three regimes which correspond to simultaneous water filling that depend on the interference gains: a) complete spectral overlap, b) partial spectral overlap, and c) spectral segregation. These imply that the transmit covariance matrices will be white in regions of both overlap and segregation, but not necessarily white overall. We also consider performance as a function of interference gain and show that complete spectral overlap is a strongly suboptimal solution over a wide range of gains. Overall, our results suggest that for strong mutual interference, an effort should be made to do joint decoding over receivers since such collaboration can provide large capacity increases. For moderate interference, distributed and/or centralized conflict resolution algorithms would be most effective since more complex collaborative methods do not afford much improvement and strictly greedy methods such as water filling perform poorly, while for weak interference a laissez faire approach seems reasonable.
\end{abstract}

\section{Index Terms}

Interference channel, multibase systems, MIMO, water filling, capacity.

This paper was presented in part at the GLOBECOM 2003 and 2004 conferences.

O. Popescu was with the Wireless Information Network Laboratory (WINLAB), Rutgers University, and is currently with the Wireless Communications Research Laboratory (WiCoRe), the University of Texas at Dallas, 2601 N. Floyd Road, Richardson, TX 75083-0688, (email: otilia.popescu@utdallas.edu).

D. C. Popescu is with the Department of Electrical Engineering, the University of Texas at San Antonio, 6900 N Loop 1604 W, San Antonio, TX 78249-0669 (email: dimitrie.popescu@utsa.edu).

C. Rose is with the Wireless Information Network Laboratory (WINLAB), Rutgers University, 73 Brett Rd., Piscataway, NJ 08854-8060 (email: crose@ winlab.rutgers.edu). 


\section{INTRODUCTION}

In unlicensed bands, spectrum is shared by all transmitters and receivers, and decoding information from a given transmitter by the intended receiver must cope with interference from "alien" transmitters. Under certain conditions (such as co-located receivers, or receivers connected to a common high speed backbone network) a wireless communication system can be modeled as a multiple access channel [13, p. 388], for which a vast body of literature offers numerous methods of decoding information in the presence of interference [16], [19], [27], [28], [39], [40]. That is, if receivers are allowed to collaborate, then they can be regarded as one large receiver with multiple inputs and multiple outputs (MIMO), and results established for MIMO systems pertain [17], [28].

However, when no cooperation among receivers is assumed, information from a given transmitter must be decoded at its intended receiver in the presence of interference from other transmitters. This is an instance of the interference channel [13, p. 382] whose complete characterization is still an open problem. We note that an early formulation of the interference channel problem is due to Shannon [37], and was followed by results obtained decades later by Ahlswede [1], Carleial [8]-[10], Sato [34]-[36], Han and Kobayashi [18], and Costa [12]. More recent results have been obtained in the context of MIMO systems with multiple transmitters that interfere with each other by Bengtsson [3] and Blum [5].

Nonetheless, mutual interference is a fact of life in wireless systems and especially in unlicensed bands. Thus, research into practical systems has cast the mutual interference problem

in a competitive context where "opposing" systems seek to maximize their performance. For instance, $\mathrm{Yu}$ et al. [41], [42] define a non-cooperative game in which transmitters compete for data rates, and each transmitter's objective is greedy performance maximization regardless of other transmitters in the system. From this perspective it is shown that for a system with two transmitters and receivers that do not collaborate, the individual performance of both transmitters is optimized by a simultaneous water filling distribution of transmitted powers [41], [42], which may be achieved by application of the iterative water filling procedure established originally in [43]. According to $\mathrm{Yu}$ et al. the simultaneous water filling point is a Nash equilibrium solution for the system [41], [42], and in general multiple Nash equilibria are possible for a given system. We note that sometimes a socially optimal solution may not be a Nash equilibrium [15, p. 18].

In our paper we consider a similar setup as Yu et al. [41], [42] consisting of a system with 
two transmitters with non-collaborating receivers. Similar to [41], [42] we assume that the two mutually interfering transmitters have non-cooperative coding strategies such that interference cancellation can not be performed at the receivers. This is slightly different than the traditional information-theoretic approach of the interference channel problem, and in this approach, greedy capacity optimization by individual transmitters through various algorithms (like iterative water filling [41]-[43] or greedy interference avoidance [24]-[26]) converges to simultaneous water filling fixed points. We investigate the structure of possible simultaneous water filling fixed points, and derive a relationship between the spatial distribution of the transmitters and receivers (roughly characterized by their corresponding gains) and the set of potential simultaneous water filling fixed points. We also define socially optimum points for such systems, and determine when these correspond to simultaneous water filling.

The paper is organized as follows. We define the problem formally in Section II. In Section III we analyze simultaneous water filling solutions and derive the spectral structures of mutual water filling points in Section IV. In Section V we assume Gaussian signaling by both transmitters and define/illustrate the simultaneous water filling region for a symmetric system. We also introduce the notion of collective capacity (in lieu of the usual information theoretic "sum capacity") as a global performance measure. We discuss weak/moderate and strong interference in Sections V-A and V-B respectively, focusing on optimum system performance and when simultaneous water filling is optimum. In section VI we explore different system control strategies for weak, moderate and strong mutual interference and also provide bounds on relative improvement in comparison to completely collaborative systems where receivers can pool information to decode transmitted information. We close with a summary of results in section VII.

\section{SYSTEM DESCRIPTION}

Consider a wireless system with two transmitters and two receivers as depicted in FIGURE 1, which is similar to those treated in related work by Carleial [8], [10], Costa [12], and Sato [36], as well as in the recent work by $\mathrm{Yu}$ [41], [42]. The two transmitters and receivers coexist in an arbitrary real-valued signal space of dimension $N$ implied by finite common bandwidth and signaling interval constraints [21], but we do not assume the same representation for the signal space in terms of a single set of orthonormal basis functions for both transmitter-receiver pairs. Rather, we assume that each transmitter uses a different set of orthonormal basis functions for 
the signal space, and denote the two sets of orthonormal basis functions by

$$
F(t)=\left[\begin{array}{c}
f_{1}(t) \\
\vdots \\
f_{n}(t) \\
\vdots \\
f_{N}(t)
\end{array}\right] \text { and } H(t)=\left[\begin{array}{c}
h_{1}(t) \\
\vdots \\
h_{n}(t) \\
\vdots \\
h_{N}(t)
\end{array}\right]
$$

The use of two sets of basis functions by the two transmitters may be due to the fact that each of the transmitters may use a different wireless standard which is a reasonable assumption in the case of mutually interfering transmitters operating in unlicensed bands. Nevertheless, since both $F(t)$ and $H(t)$ span the same signal space, they can be related by a linear transformation represented by an $N \times N$ unitary matrix $\mathbf{U}$, such that $F(t)=\mathbf{U} H(t)$ and $H(t)=\mathbf{U}^{\top} F(t)$. We note that knowledge of this matrix is not required, since - as it will be seen in Section III - it is not relevant for our analysis of simultaneous water filling points.

We assume synchronization between transmitters at both receivers, and frequency flat communication channels characterized only by scalar gains. The gain between a given transmitter and its intended receiver is normalized to 1 , and we denote by $g_{1}$ the interference gain corresponding to transmitter 1's signal at receiver 2 and $g_{2}$ the interference gain corresponding to transmitter 2's signal at receiver 1 .

Letting $x_{\ell}(t)$ be the signal sent by transmitter $\ell=1,2$, the signals at the two receivers are

$$
\begin{aligned}
& r_{1}(t)=x_{1}(t)+\sqrt{g_{2}} x_{2}(t)+n_{1}(t) \\
& r_{2}(t)=\sqrt{g_{1}} x_{1}(t)+x_{2}(t)+n_{2}(t)
\end{aligned}
$$

where $n_{\ell}(t)$ is the additive white Gaussian noise corrupting the signals at receiver $\ell=1,2$. For simplicity we assume that noise characteristics are the same at both receivers, with zero mean and power spectral density $\eta_{0}$. We note that the signals from the two transmitters can be written in terms of the basis functions in equation (1) as

$$
\begin{gathered}
x_{1}(t)=F^{\top}(t) \mathbf{x}_{1}=H^{\top}(t) \mathbf{U}^{\top} \mathbf{x}_{1} \\
x_{2}(t)=H^{\top}(t) \mathbf{x}_{2}=F^{\top}(t) \mathbf{U} \mathbf{x}_{2}
\end{gathered}
$$

with $\mathrm{x}_{1}$ and $\mathrm{x}_{2}$ being the corresponding signal vectors. The transmitted power spectral density for transmitter $\ell$ is $S_{x_{\ell}}(f)$ and the corresponding transmitted power is

$$
P_{\ell}=E\left[x_{\ell}^{2}(t)\right]=\int_{-\infty}^{\infty} S_{x_{\ell}}(f) d f \quad \ell=1,2
$$


By projecting the received signals in equation (2) onto the basis functions $F(t)$ and $G(t)$ at receiver 1 and receiver 2 respectively, we obtain the corresponding signal vectors

$$
\begin{gathered}
\mathbf{r}_{1}=\mathbf{x}_{1}+\sqrt{g_{2}} \mathbf{U} \mathbf{x}_{2}+\mathbf{n}_{1} \\
\mathbf{r}_{2}=\sqrt{g_{1}} \mathbf{U}^{\top} \mathbf{x}_{1}+\mathbf{x}_{2}+\mathbf{n}_{2}
\end{gathered}
$$

with $\mathbf{x}_{1}$ and $\mathbf{x}_{2}$ the signal vectors sent by the two transmitters, and $\mathbf{n}_{1}$ and $\mathbf{n}_{2}$ white Gaussian noise vectors with covariance matrices $E\left[\mathbf{n}_{1} \mathbf{n}_{1}^{\top}\right]=E\left[\mathbf{n}_{2} \mathbf{n}_{2}^{\top}\right]=\eta_{0} \mathbf{I}_{N}$. Let $\mathbf{X}_{\ell}=E\left[\mathbf{x}_{\ell} \mathbf{x}_{\ell}^{\top}\right], \ell=1,2$, be the transmit covariance matrices of the two transmitters, with the transmitted powers

$$
P_{\ell}=E\left[\left\|\mathbf{x}_{\ell}\right\|^{2}\right]=\text { Trace }\left[\mathbf{X}_{\ell}\right] \quad \ell=1,2
$$

We assume each receiver treats interference from the other as (colored) Gaussian noise. Thus, capacities expressed in standard units of [bits/transmission] [13] can be written as [43]

$$
\begin{aligned}
& C_{1}=\frac{1}{2} \log \left|\mathbf{R}_{1}\right|-\frac{1}{2} \log \left|g_{2} \mathbf{U X}_{2} \mathbf{U}^{\top}+\eta_{0} \mathbf{I}_{N}\right| \\
& C_{2}=\frac{1}{2} \log \left|\mathbf{R}_{2}\right|-\frac{1}{2} \log \left|g_{1} \mathbf{U}^{\top} \mathbf{X}_{1} \mathbf{U}+\eta_{0} \mathbf{I}_{N}\right|
\end{aligned}
$$

where $\log (\cdot)$ denotes the base $2 \operatorname{logarithm}$, and $\mathbf{R}_{1}$ and $\mathbf{R}_{2}$ are the correlation matrices at each receiver expressed as

$$
\begin{aligned}
& \mathbf{R}_{1}=E\left[\mathbf{r}_{1} \mathbf{r}_{1}^{\top}\right]=\mathbf{X}_{1}+g_{2} \mathbf{U X}_{2} \mathbf{U}^{\top}+\eta_{0} \mathbf{I}_{N} \\
& \mathbf{R}_{2}=E\left[\mathbf{r}_{2} \mathbf{r}_{2}^{\top}\right]=g_{1} \mathbf{U}^{\top} \mathbf{X}_{1} \mathbf{U}+\mathbf{X}_{2}+\eta_{0} \mathbf{I}_{N}
\end{aligned}
$$

In this framework, capacity can be optimized by individual transmitters through various algorithms (like iterative water filling [41]-[43] or greedy interference avoidance [24]-[26]) which converge to simultaneous water filling fixed points where the spectrum of the transmit covariance matrix of one user water fills over the spectrum of its corresponding interference-plusnoise covariance matrix. In our paper we will not focus on a specific algorithm by which such fixed points are reached, and note that these have been discussed in [25], [26], [41], [42]. Rather, we concentrate on the spectral structure of user transmit covariance matrices at simultaneous water filling fixed points and their performance.

In the following section we will show that the greedy capacity optimization implied by simultaneous water filling leads to transmit covariance matrices that imply a diagonal structure on the correlation matrices of the received signals at the two receivers, which is similar to classical water filling solutions. Then, we will show that unlike classical water filling spectra 
for single or multiple transmitters with a single receiver, mutually water-filled spectra are in this case constrained to three different classes - completely overlapped, segregated, and partially overlapped. Furthermore, the spectrum in each region must be white although the spectra $\mathbf{X}_{i}$ are not necessarily white themselves.

\section{Simultaneous Water Filling}

Simultaneous water filling is formally defined by Yu [41] as a generalization of the single-user water filling to multiple user scenarios. For the considered system with two mutually interfering transmitters described in the previous section, a simultaneous water filling point is reached when one transmitter maximizes its corresponding capacity by regarding the other transmitter's interfering signal as Gaussian noise and distributes its transmit energy over the signal space according to a single-user water filling scheme [13, p. 253], and vice versa. As noted in the previous section, simultaneous water filling for the considered system with two transmitters and receivers can be achieved by using algorithms like iterative water filling [41], [42] or interference avoidance [25], [26], which are not discussed here since they lie outside the scope of the paper. We note that these algorithms yield the transmit covariance matrices $\mathbf{X}_{1}$ and $\mathbf{X}_{2}$ whose spectral structure will be investigated in the subsequent sections of the paper.

The main result of this section is to show that at a simultaneous water filling point the two transmit covariance matrices have the same eigenvectors from the perspective of each receiver, that is, at each receiver the eigenspaces of the signals from the two transmitters are aligned. We state this result formally as a theorem:

Theorem 1 (Alignment): At a simultaneous water filling point, at a given receiver, the signals from the intended user and the interfering user are aligned along the same eigenvectors.

Proof: Let us express the transmit covariance matrices of the two transmitters in terms of their eigenvectors and eigenvalues using the spectral factorization theorem [20, p. 104], [38, p. 296] as

$$
\begin{aligned}
& \mathbf{X}_{1}=\sum_{i=1}^{N} \alpha_{i} \psi_{i} \psi_{i}^{\top}=\mathbf{\Psi} \mathbf{A} \boldsymbol{\Psi}^{\top} \\
& \mathbf{X}_{2}=\sum_{i=1}^{N} \beta_{i} \phi_{i} \phi_{i}^{\top}=\mathbf{\Phi B} \boldsymbol{\Phi}^{\top}
\end{aligned}
$$

where $\boldsymbol{\Psi}$ and $\boldsymbol{\Phi}$ are the matrices of eigenvectors $\left\{\psi_{i}\right\}_{i=1}^{N}$ and $\left\{\phi_{i}\right\}_{i=1}^{N}$ for $\mathbf{X}_{1}$ and $\mathbf{X}_{2}$, respectively. $\mathbf{A}$ and $\mathbf{B}$ are the corresponding diagonal eigenvalue matrices, with eigenvalues $\alpha_{1} \geq \ldots \geq \alpha_{N}$ 
and $\beta_{1} \leq \ldots \leq \beta_{N}$, in decreasing and increasing order of their magnitudes, respectively. We note that eigenvalues are real and non-negative since the $\mathbf{X}_{\ell}$ are covariance matrices, and are therefore positive semidefinte.

From the perspective of a given transmitter, the other transmitter is Gaussian interference, and, in order to achieve minimum mutual interference, at a simultaneous water filling point the eigenvectors of its transmit covariance matrix must align with the eigenvectors of the interferenceplus-noise covariance matrix [32], [33], [41], [43]. Therefore, at a simultaneous water filling point, at receiver 1 the transmit covariance matrix $\mathbf{X}_{1}$ of transmitter 1 and interference-plusnoise covariance matrix $g_{2} \mathbf{U X}_{2} \mathbf{U}^{\top}+\eta_{0} \mathbf{I}_{N}$ will have the same eigenvectors specified by $\Psi$. This implies that $\Psi$ diagonalizes the interference-plus-noise covariance matrix and we can write

$$
\underbrace{\boldsymbol{\Psi}^{\top}\left(g_{2} \mathbf{U X}_{2} \mathbf{U}^{\top}+\eta_{0} \mathbf{I}_{N}\right) \boldsymbol{\Psi}}_{\text {diagonal }}=g_{2} \boldsymbol{\Psi}^{\top} \mathbf{U X}_{2} \mathbf{U}^{\top} \boldsymbol{\Psi}+\underbrace{\eta_{0} \mathbf{I}_{N}}_{\text {diagonal }}
$$

We note that since the left hand side is a diagonal matrix, and the second term in the right hand side is also a diagonal matrix, then the first term in the right hand side must be a diagonal matrix as well, which implies that $\mathbf{U X}_{2} \mathbf{U}^{\top}$ is diagonalized by the same set of eigenvectors $\Psi$ as $\mathbf{X}_{1}$. Thus, at a simultaneous water filling point, at a receiver 1 the signals from the intended transmitter 1 and the interfering transmitter 2 are aligned along the same eigenvectors specified by $\Psi$. We can show similarly that at recevier 2 the eigenvectors of $\mathbf{U}^{\top} \mathbf{X}_{1} \mathbf{U}$ and $\mathbf{X}_{2}$ are the same and equal to $\Phi$.

Corollary 1 (Diagonal Structure): At a simultaneous water filling point, at a given receiver, the received signal correlation matrix is diagonal.

Proof: Multiplying the received signal correlation matrices $\mathbf{R}_{1}$ and $\mathbf{R}_{2}$ in equation (8) with their corresponding eigenvector matrices $\Psi$ and $\Phi$, and using the alignment result in Theorem 1 implies

$$
\begin{aligned}
& \boldsymbol{\Lambda}_{1}=\boldsymbol{\Psi}^{\top} \mathbf{R}_{1} \boldsymbol{\Psi}=\mathbf{A}+g_{2} \mathbf{B}+\eta_{0} \mathbf{I}_{N} \\
& \boldsymbol{\Lambda}_{2}=\boldsymbol{\Phi}^{\top} \mathbf{R}_{2} \boldsymbol{\Phi}=\mathbf{B}+g_{1} \mathbf{A}+\eta_{0} \mathbf{I}_{N}
\end{aligned}
$$

which shows that the $\Lambda_{i}$ are diagonal matrices.

We conclude this section by noting that the eigenvalues of the transmit covariance matrix of a given transmitter must also satisfy a water filling distribution [13, p. 253] over those eigenvectors 
of the corresponding interference-plus-noise covariance matrix with minimum eigenvalues.

\section{Simultaneous Water Filling Structures}

As mentioned in the previous section, a simultaneous water filling point can be obtained by application of various algorithms, which will yield a set of transmit covariance matrices $\mathbf{X}_{1}$ and $\mathbf{X}_{2}$ corresponding to the signals sent by the two transmitters. In this section, we discuss the possible spectral structures that correspond to these covariance matrices. We note that in light of the diagonal structure result proved in the previous section, we focus the discussion on the diagonal eigenvalue matrices $\mathbf{A}$ and $\mathbf{B}$ corresponding to the two transmit covariance matrices $\mathbf{X}_{1}$ and $\mathbf{X}_{2}$, which are the ones that determine the spectrum of the simultaneous water filling structures.

The three possible spectral structures for simultaneously water-filled transmit covariance matrices are:

1) Complete overlap of the two spectra, when all dimensions of the signal space are utilized by both transmitters.

2) Incomplete overlap of the two spectra, when only a subset of signal dimensions is shared, leaving some dimensions for exclusive use by a single transmitter.

3) No overlap of the two spectra, when transmitters place their signal energy in orthogonal signal subspaces.

We derive the properties of such spectra in the following three sections.

\section{A. Complete Overlap Between Transmitted Spectra}

Suppose the transmit covariance matrices for both transmitters span the entire signal space, which corresponds to complete overlap in signal space between the transmitted spectra. Water filling requires that eigenvalue matrices in equation (11) be scaled identity matrices, that is

$$
\begin{aligned}
& \mathbf{A}+g_{2} \mathbf{B}+\eta_{0} \mathbf{I}_{N}=c_{1} \mathbf{I}_{N} \\
& \mathbf{B}+g_{1} \mathbf{A}+\eta_{0} \mathbf{I}_{N}=c_{2} \mathbf{I}_{N}
\end{aligned}
$$

where $c_{1}$ and $c_{2}$ are the "watermarks" corresponding to the two transmitters, and are obtained using the trace constraints in equation (6) as

$$
c_{1}=\frac{P_{1}+g_{2} P_{2}}{N}+\eta_{0} \quad \text { and } \quad c_{2}=\frac{P_{2}+g_{1} P_{1}}{N}+\eta_{0}
$$


With the exception of the case $g_{1}=g_{2}=1$ when the two matrix equations implied by (12) and (13) are identical, the solution of the system of matrix equations (12) is

$$
\mathbf{A}=\frac{P_{1}}{N} \mathbf{I}_{N} \quad \text { and } \quad \mathbf{B}=\frac{P_{2}}{N} \mathbf{I}_{N}
$$

Since all eigenvalues of transmit covariance matrices for both transmitters are equal in this case, the water filling structure is also unique and implies that the transmit covariance matrices corresponding to the two transmitters must be scaled identity matrices.

When $g_{1}=g_{2}=1$ multiple solutions are possible such that these satisfy the undetermined matrix equation

$$
\mathbf{A}+\mathbf{B}+\eta_{0} \mathbf{I}_{N}=c \mathbf{I}_{N} \quad \text { with } \quad c=\frac{P_{1}+P_{2}}{N}+\eta_{0}
$$

We conclude this section by noting that complete spectral overlap is possible for any values of the interference gains $g_{1}$ and $g_{2}$, and is unique except for the case of $g_{1}=g_{2}=1$ when many solutions are possible.

\section{B. Incomplete Overlap Between Transmitted Spectra}

Now suppose that transmitter $i \in\{1,2\}$ covariance matrix at a simultaneous water filling point spans $k_{i}$ dimensions of the signal space, such that $k_{1}+k_{2}>N$ and the two spectra overlap in $k_{1}+k_{2}-N$ dimensions. We note that $k_{i}$ coincides with the rank of transmitter $i$ covariance matrix, and is fixed once the simultaneous water filling point is reached.

From transmitter 1's perspective, water filling implies that the first $k_{1}$ values of $\Lambda_{1}$ in equation (11) must be identical and equal to the "water level" $\mathcal{L}_{1}$ in transmitter 1 's subspace. This water level is determined from the trace constraints as

$$
\begin{aligned}
\mathcal{L}_{1}= & \frac{\operatorname{Trace}\left[\mathbf{X}_{1}\right]+g_{2} \sum_{i=1}^{k_{1}} \beta_{i}}{k_{1}}+\eta_{0} \\
= & \frac{P_{1}+g_{2} \sum_{i=1}^{k_{1}} \beta_{i}}{k_{1}}+\eta_{0}
\end{aligned}
$$

Equation (16) along with the water filling condition [13, p. 253] implies that the eigenvalues of the transmit covariance matrix of transmitter 1 can be expressed as

$$
\alpha_{i}=\left(\mathcal{L}_{1}-g_{2} \beta_{i}-\eta_{0}\right)^{+} \quad i=1, \ldots, N
$$


where the notation $(x)^{+}$denotes the positive part of $x[13, \mathrm{p} .253]$ that is $(x)^{+}=x$ if $x \geq 0$ and $(x)^{+}=0$ if $x<0$. This implies that

$$
\alpha_{i}=\left\{\begin{array}{cl}
\mathcal{L}_{1}-g_{2} \beta_{i}-\eta_{0} & \text { for } i=1, \ldots, k_{1} \\
0 & \text { for } i=k_{1}+1, \ldots, N
\end{array}\right.
$$

and $\mathbf{X}_{1}$ is expressed only in terms of its first $k_{1}$ eigenvectors as

$$
\mathbf{X}_{1}=\sum_{i=1}^{k_{1}}\left(\mathcal{L}_{1}-g_{2} \beta_{i}-\eta_{0}\right) \phi_{i} \phi_{i}^{\top}
$$

Similarly, from the perspective of the transmit covariance matrix of transmitter 2, water filling implies that the last $k_{2}$ values of $\boldsymbol{\Lambda}_{2}$ must be identical. Proceeding as for transmitter 1 we have

$$
\mathcal{L}_{2}=\frac{P_{2}+g_{1} \sum_{i=N-k_{2}+1}^{N} \alpha_{i}}{k_{2}}+\eta_{0}
$$

with eigenvalues of the transmit covariance matrix of transmitter 2 expressed as

$$
\beta_{j}=\left(\mathcal{L}_{2}-g_{1} \alpha_{j}-\eta_{0}\right)^{+} \quad j=1, \ldots, N
$$

or

$$
\beta_{j}=\left\{\begin{array}{cl}
0 & \text { for } j=1, \ldots, N-k_{2} \\
\mathcal{L}_{2}-g_{1} \alpha_{j}-\eta_{0} & \text { for } \quad j=N-k_{2}+1, \ldots, N
\end{array}\right.
$$

The transmit covariance matrix of transmitter 2 is written then only in terms of its last $k_{2}$ eigenvectors as

$$
\mathbf{X}_{2}=\sum_{j=N-k_{2}+1}^{N}\left(\mathcal{L}_{2}-g_{1} \alpha_{j}-\eta_{0}\right) \phi_{j} \phi_{j}^{\top}
$$

We note that incomplete overlap between the two spectra in signal space does not imply a unique solution since the dimensions $\left(k_{1}, k_{2}\right)$ of the two subspaces spanned by the transmit covariance matrices of the two transmitters are not unique. These various solutions may be obtained by using different initializations in the algorithms that generate the transmit covarince matrices $\mathbf{X}_{1}$ and $\mathbf{X}_{2}$.

At this point we distinguish two cases of incomplete overlap between the two transmitter spectra:

1) Partial overlap with $k_{1}, k_{2}<N$, where no transmit covariance matrix spans the entire signal space. 
2) Nesting partial overlap with $k_{1}=N$ and $k_{2}<N$, where the transmit covariance matrix of transmitter 1 spans the entire signal space, and the spectrum of the transmit covariance matrix of transmitter 2 appears "nested" with it).

1) Partial Overlap: $k_{1}, k_{2}<N$ : In this case transmitter 1 's signal occupies alone $N-k_{2}$ dimensions of the signal space, transmitter 2's signal occupies alone $N-k_{1}$ dimensions of the signal space, and the two signals overlap in $k_{1}+k_{2}-N$ dimensions. The watermark from the perspective of transmitter 1 (transmitter 2) is determined by its largest eigenvalue, $\alpha_{1}\left(\beta_{N}\right)$, and the water filling structure imposes the following constraints:

$$
\begin{gathered}
\alpha_{1}=\alpha_{2}=\ldots=\alpha_{N-k_{2}}= \\
=\alpha_{N-k_{2}+1}+g_{2} \beta_{N-k_{2}+1}=\ldots=\alpha_{k_{1}}+g_{2} \beta_{k_{1}} \\
\beta_{N}=\beta_{N-1}=\ldots=\beta_{k_{1}+1}= \\
=g_{1} \alpha_{k_{1}}+\beta_{k_{1}}=\ldots=g_{1} \alpha_{N-k_{2}+1}+\beta_{N-k_{2}+1}
\end{gathered}
$$

In addition, the water filling condition requires also that the watermark $\mathcal{L}_{1}\left(\mathcal{L}_{2}\right)$ be always less than or equal to the interference plus noise levels on the dimensions that are not water filled [13, p. 253]. This implies the following relationships for the largest eigenvalues of the two transmit covariance matrices

$$
\alpha_{1} \leq g_{2} \beta_{N} \quad \text { and } \quad \beta_{N} \leq g_{1} \alpha_{1}
$$

which further implies that the incomplete overlap case of the water filling structure is possible only if the interference gains satisfy

$$
g_{1} g_{2} \geq 1
$$

By solving the system of equations (24) - (25) one can see that when $g_{1} g_{2}>1$, all the eigenvalues can be written in terms of the largest eigenvalues $\alpha_{1}$ and $\beta_{N}$ as

$$
\left\{\begin{array}{l}
\alpha_{1}=\alpha_{2}=\ldots=\alpha_{N-k_{2}} \\
\alpha_{N-k_{2}+1}=\ldots=\alpha_{k_{1}}=\frac{g_{2} \beta_{N}-\alpha_{1}}{g_{1} g_{2}-1} \\
\alpha_{k_{1}+1}=\ldots=\alpha_{N}=0
\end{array}\right.
$$

and

$$
\left\{\begin{array}{l}
\beta_{N}=\beta_{N-1}=\ldots=\beta_{k_{1}+1} \\
\beta_{N-k_{2}+1}=\ldots=\beta_{k_{1}}=\frac{g_{1} \alpha_{1}-\beta_{N}}{g_{1} g_{2}-1} \\
\beta_{1}=\ldots=\beta_{N-k_{2}}=0
\end{array}\right.
$$


Thus, when $g_{1} g_{2}>1$ the eigenvalues of the transmit covariance matrix of each transmitter can have only two nonzero values: one for the unshared dimensions of the signal space and another for the shared dimensions of the signal space.

When $g_{1} g_{2}=1$, we can denote

$$
g_{1}=g \quad \text { and } \quad g_{2}=\frac{1}{g}
$$

such that the two systems of equations (24) - (25) become identical

$$
g \alpha_{n}+\beta_{n}=g \alpha_{1}=\beta_{N} \quad n=N-k_{2}+1, \ldots, k_{1}
$$

In this case it is no longer possible to find expressions for each eigenvalue in terms of the largest eigenvalues $\alpha_{1}$ and $\beta_{N}$ as before, and theoretically any values that satisfy equation (31) will also satisfy the required water filling conditions.

2) Nested Partial Overlap: $k_{1}=N$ and $k_{2}<N$ : In this case we assume with no loss of generality that transmitter 1's signal occupies all $N$ dimensions of the signal space while transmitter 2's signal occupies only $k_{2}$ dimensions of the signal space, such that the two signals overlap in the $k_{2}$ dimensions of the signal space that are occupied by transmitter 2 . Thus, in this case the transmit covariance matrix of transmitter 1 spans the entire signal space and the transmit covariance matrix of transmitter 2 spans only a proper subspace. That is, transmitter 2's signal space is completely contained (or nested) in transmitter 1's signal space. The derivation in previous section applies with minor changes, and the water filling conditions in equation (26) imply in this case that

$$
g_{2} \beta_{N}+\alpha_{N}=\alpha_{1} \quad \text { and } \quad \beta_{N}+g_{1} \alpha_{N} \leq g_{1} \alpha_{1}
$$

which corresponds also to $g_{1} g_{2} \geq 1$.

When $g_{1} g_{2}>1$ the eigenvalues of the transmit covariance matrix of the first transmitter are given in this case by

$$
\left\{\begin{array}{l}
\alpha_{1}=\alpha_{2}=\ldots=\alpha_{N-k_{2}} \\
\alpha_{N-k_{2}+1}=\ldots=\alpha_{N}=\frac{g_{2} \beta_{N}-\alpha_{1}}{g_{1} g_{2}-1}
\end{array}\right.
$$

while those of the transmit covariance matrix of the second user are given by

$$
\left\{\begin{array}{l}
\beta_{N}=\beta_{N-1}=\ldots=\beta_{N-k_{2}+1} \\
\beta_{1}=\ldots=\beta_{N-k_{2}}=0
\end{array}\right.
$$


When $g_{1} g_{2}=1$ the eigenvalue expressions for the overlapped dimensions $N-k_{2}+1, \ldots, N$ cannot be determined uniquely, and similar to the previous case, any values that satisfy the water filling conditions are possible.

\section{No Overlap: $k_{1}+k_{2}=N$}

Finally, suppose that the transmit covariance matrices of the two transmitters span orthogonal subspaces of the signal space at a simultaneously water filling point, that is, assume that transmitter 1 signals in a subspace of dimension $k$ and transmitter 2 signals in the remaining $N-k$ dimensions of the signal space. We note that $k$ coincides with the rank of transmitter 1 covariance matrix, and is fixed once the simultaneous water filling point is reached.

Using the trace constraints in equation (6) we obtain

$$
\left\{\begin{array}{l}
\alpha_{1}=\alpha_{2}=\ldots=\alpha_{k}=\frac{P_{1}}{k} \\
\alpha_{1} \leq g_{2} \beta_{N}
\end{array}\right.
$$

and

$$
\left\{\begin{array}{l}
\beta_{k+1}=\ldots=\beta_{N-1}=\beta_{N}=\frac{P_{2}}{N-k} \\
g_{1} \alpha_{1} \geq \beta_{N}
\end{array}\right.
$$

which implies that

$$
g_{1} g_{2} \geq 1
$$

and

$$
\frac{P_{1}}{P_{1}+P_{2} g_{2}} \leq \frac{k}{N} \leq \frac{g_{1} P_{1}}{g_{1} P_{1}+P_{2}}
$$

Thus, in general the solution for $k$ is not unique, and can be any integer in the interval

$$
k \in\left[\frac{P_{1}}{P_{1}+P_{2} g_{2}} N, \frac{g_{1} P_{1}}{g_{1} P_{1}+P_{2}} N\right]
$$

We note that as the interference gains increase the range of $k$ expands since the lower limit decreases when $g_{2}$ increases and the upper limit increases when $g_{1}$ increases. We also note that for the particular case corresponding to $g_{1} g_{2}=1$ there may be no solution for $k$ in the discrete representation considered. This is because when $g_{1} g_{2}=1$ we can replace for example $g_{2}=1 / g_{1}$ in the lower bound so that this becomes equal to the upper bound or vice-versa, and if the resulting number is not an integer there will be no solution for $k$ in this case. Nevertheless, as 
$N \rightarrow \infty$ there will always exist an integer upper/lower bound in equation (39) when $g_{1} g_{2}=1$ which implies a unique solution for $k$.

We conclude this section by formally summarizing the relationship between number of possible simultaneous water filling solutions and the interference gain values $g_{1}$ and $g_{2}$ in the following theorem:

Theorem 2 (Overlap): The interference gain product $g_{1} g_{2}$ arbitrates the types of spectral overlap possible with mutually water-filled spectra.

1) $g_{1} g_{2} \geq 1$ : the transmitted spectra may overlap, and must be "white" in both the overlapped and non-overlapped regions. There can be many such sets of spectra.

2) $g_{1} g_{2}<1$ : each transmitted spectrum must be white and span the entire signal space - a unique solution.

Proof: The first statement of the theorem is a rehash of the observations presented in sections IV-B and IV-C where we have shown that incomplete overlap, as well as separation of transmitted spectra correspond to $g_{1} g_{2} \geq 1$. For the second statement, we note that when $g_{1} g_{2}<1$ neither incomplete spectral overlap, nor spectral separation are possible since the interference gain product is less than 1 in this case. Thus, when $g_{1} g_{2}<1$ only complete spectral overlap is possible. This solution is unique since in this case all dimensions of the signal space are spanned by both transmit covariance matrices.

Using distance as a proxy for interference gain values $\left\{g_{1}, g_{2}\right\}$ the fixed point classifications of Theorem 2 are represented pictorially in Table I.

\section{The Simultaneous Water Filling Region}

We define the simultaneous water filling region as the set of all possible capacity pairs $\left(C_{1}, C_{2}\right)$ under the assumption of Gaussian signaling by each transmitter. We note that the simultaneous water filling region depends on the interference gain values $g_{1}$ and $g_{2}$ since these affect the capacity values (see equation (7)) as well as the possible simultaneous water filling structures (see Table I).

Using physical distance as a proxy for the interference gain value (i.e., farther away implies lower value) and equal transmitted powers, the scenario $g_{1} g_{2}<1$ corresponds roughly to "weak/moderate interference", in which the distance from at least one transmitter to its associated receiver is smaller than its distance to the "alien" receiver - i.e., the interfering signal at the 
alien receiver is weaker than the desired signal. The scenario $g_{1} g_{2}>1$ roughly corresponds to "strong interference" where a given transmitter is close to the "alien" receiver associated to the other transmitter, and interferes strongly with its transmission.

To illustrate the simultaneous water filling region and its dependence on interference gain values, we consider a particular example of a symmetric system with $N=100$ signal space dimensions, gains $g_{1}=g_{2}=g$, transmitted powers $P_{1}=P_{2}=10$, and background noise level $\eta_{0}=0.01$. For these numerical values the signal-to-noise ratio (SNR) at the receiver

$$
\rho=\frac{P}{N \eta_{0}}
$$

is $\rho=10$ or $10 \mathrm{~dB}$. FIGURE 2 shows various simultaneous water filling regions corresponding to different values of $g$ for the considered system.

Referring to FIGURE 2, our previous analysis indicates that for $g<1$ the simultaneous water filling region consists of a single point, and this is shown for several values of $g<1$ in the upper left plot of FIGURE 2. We note that as $g$ increases getting closer to 1 the two capacities decrease, and the simultaneous water filling point gets closer to the origin. We also note that in the case of $g<1$ we distinguish a truly weak interference scenario (e.g. points with $g<0.18$ in the figure $^{1}$ ) for which simultaneous water filling implies good system performance with capacities relatively close to those in the no interference case, as well as a moderate interference scenario for which performance of simultaneous water filling degrades with capacities in this case far away from those in the no interference case.

The case of $g \geq 1$ corresponds to strong interference, and is illustrated in the remaining three plots of FIGURE 2. In this case the simultaneous water filling region consists of multiple points which can be classified in three categories according to the three types of spectral overlap discussed in the previous section:

- A most interior point corresponding to complete overlap of the spectra of the two transmit covariance matrices at which both capacities have minimum values since transmitters interfere in all dimensions of the signal space at this point. The most interior point moves closer to the origin as $g$ increases showing that capacities decrease due to the increased interference from the "alien" transmitter.

\footnotetext{
${ }^{1}$ The value of $g=0.18$ was obtained using equation (51) in Section V-A.
} 
- An outer border containing all points that correspond to spectral separation of the two transmit covariance matrices for which the two transmitters do not interfere with each other. As $g$ increases, the outer border expands from a single point in the case $g=1$ to more points that correspond to more potential partitions of the signal space between the two transmitters.

- An interior region containing all points between the most interior point and the outer border that correspond to incomplete spectral overlap for which transmitters interfere only in some dimensions of the signal space.

We note that the points that make up the simultaneous water filling region have capacity values which are always less than the capacities without interference, which can be obtained by setting the interference gains equal to zero. When $g=0$, signals sent by the two transmitters do not interfere with each other and the corresponding capacities are

$$
C_{\ell}=\frac{N}{2} \log \left(1+\frac{P_{\ell}}{N \eta_{0}}\right) \quad \ell=1,2
$$

and the point implied by $\left(C_{1}, C_{2}\right)-(1.73,1.73)$ on the plots in FIGURE 2 - is always above the simultaneous water filling region. According to Carleial [8] this point is achievable in the case of strong interference by subtracting interference from the desired signal and decoding it as in the absence of interference, but it does not correspond to a simultaneous water filling point since it lies outside of the simultaneous water filling region.

To evaluate the performance of the system overall, we introduce the sum of capacities

$$
\mathcal{C}=C_{1}+C_{2}
$$

which we call the collective capacity in order to distinguish it from the information-theoretic sum capacity used when receivers can collaborate [27], [28]. This measure is useful in the case $g_{1} g_{2} \geq 1$ (where multiple simultaneous water filling points are possible) to evaluate which point is a most desirable from a global perspective. It is also useful in the case $g_{1} g_{2}<1$ (when the simultaneous water filling point is unique) to compare it with other spectral partitions which may be more desirable for the system.

We define the social optimum as the point for which the collective capacity $\mathcal{C}$ is maximized. We note that, when $g_{1} g_{2}=1$ the outer border of the simultaneous water filling region consists of a single point where both transmitters maximize their capacities, and which corresponds to 
complete spectral separation in signal space. At this point both individual and collective interests are satisfied since the collective capacity $\mathcal{C}$ is also maximized at this point, and we note that complete spectral separation corresponds to a social optimum in this case.

What is perhaps most interesting about the montage of FIGURE 2 is that from a system design perspective, the weak and strong interference cases are least problematic. For weak interference (shown in upper left plot of FIGURE 2), mutual interference levels are comparable to or below the noise floor so mutually water-filled solutions will provide good system performance with capacities relatively close to those in the no interference case. For strong interference (shown in the remaining three plots of FIGURE 2 for $g=1,2,100)$ there exists a mutual water filling solution which is also optimum from a collective capacity standpoint. This solution corresponds to signal space partitioning between transmitters, and is situated on the outer border of the simultaneous water filling region as shown in the corresponding plots of FIGURE 2. In addition, in the case of strong interference all the other points on the outer border of the simultaneous water filling region, although suboptimal, correspond to collective capacity values close to the optimal value [25], [30]. In contrast, for moderate interference (shown in upper left plot of FIGURE 2 along with weak interference) simultaneous water filling leads to capacity values that are much below those in the no interference case, and transmitters would be better served if they agreed (or were forced) to signal in different regions of the signal space. We explore these various levels of interference in the next sections.

\section{A. Weak and Moderate Interference}

In his doctoral dissertation [41] Yu approaches the system with two mutually interfering transmitters and non-cooperating receivers from a game-theoretic perspective, and models this instant of the interference channel problem as a non-cooperative game in which the two transmitters compete for maximizing their capacities. Using this approach it is shown that when interference gains $g_{1} g_{2}<1$, simultaneous water filling represents a Nash equilibrium point of the noncooperative interference channel game [41]. In game theory, a Nash equilibrium is defined by a set of strategies such that each player's strategy is an optimal response to the other players' strategies [15, p. 11]. From this perspective, a Nash equilibrium is reached for the Gaussian interference channel game if and only if a simultaneous water filling solution is produced by both transmitters, and the optimal signaling strategy of each transmitter is to water fill the signal 
space while regarding the interfering signal of the other transmitter as noise [41]. We note that a Nash equilibrium is said to be Pareto deficient (or non-Pareto-optimal) if at least one player would do better and the other one would do no worse by switching to a different strategy [44, p. 52]. Such Nash equilibria are not necessarily efficient in that there exist cooperative strategies where both players achieve better returns. Classical examples in this sense are the Prisoner's Dilemma [44, p. 51] or tit for tat strategies [22]. For $g_{1} g_{2}<1$ we see from FIGURE 2 that capacities achieved by transmitters under simultaneous water filling decrease as interference gains increase, and we will show that the Nash equilibrium implied by simultaneous water filling in this case can be either efficient or inefficient depending upon the level of interference. Specifically, we will explore the relative efficiencies of simultaneous water filling and signal space partitioning (or segregation) under moderate and weak interference.

With simultaneous water filling each transmitter achieves capacity

$$
C_{1}^{w f}=\frac{N}{2} \log \left(1+\frac{P_{1}}{g_{2} P_{2}+N \eta_{0}}\right) \quad C_{2}^{w f}=\frac{N}{2} \log \left(1+\frac{P_{2}}{g_{1} P_{1}+N \eta_{0}}\right)
$$

and the collective capacity is

$$
\begin{aligned}
\mathcal{C}_{w f} & =C_{1}^{w f}+C_{2}^{w f} \\
& =\frac{N}{2} \log \left(1+\frac{P_{1}}{g_{2} P_{2}+N \eta_{0}}\right)+\frac{N}{2} \log \left(1+\frac{P_{2}}{g_{1} P_{1}+N \eta_{0}}\right)
\end{aligned}
$$

For simplicity let us assume a symmetric system with $P_{1}=P_{2}=P, g_{1}=g_{2}=g$, and the raw SNR $\rho$ as defined in equation (40), for which we obtain

$$
\mathcal{C}_{w f}=N \log \left(1+\frac{\rho}{\rho g+1}\right)
$$

With signal space partitioning, capacities are given by

$$
\begin{gathered}
C_{1}^{s p}=\frac{k}{2} \log \left(1+\frac{P_{1}}{k \eta_{0}}\right) \\
C_{2}^{s p}=\frac{N-k}{2} \log \left[1+\frac{P_{2}}{(N-k) \eta_{0}}\right]
\end{gathered}
$$

so the collective capacity is

$$
\begin{aligned}
\mathcal{C}_{s p} & =C_{1}^{s p}+C_{2}^{s p}=\frac{k}{2} \log \left(1+\frac{P_{1}}{k \eta_{0}}\right) \\
& +\frac{N-k}{2} \log \left[1+\frac{P_{2}}{(N-k) \eta_{0}}\right]
\end{aligned}
$$


with $k$ the number of dimensions occupied by transmitter 1 . Once again for simplicity we assume a symmetric system to obtain

$$
\mathcal{C}_{s p}=\frac{k}{2} \log \frac{\left(1+\frac{\rho}{k / N}\right)}{\left(1+\frac{\rho}{1-k / N}\right)}+\frac{N}{2} \log \left[1+\frac{\rho}{(1-k / N)}\right]
$$

which is maximized by $k=N / 2$ which implies

$$
\mathcal{C}_{s p}^{*}=\frac{N}{2} \log [1+2 \rho]
$$

We plot values $C_{1}^{s p}, C_{2}^{s p}$, and $C_{1}^{w f}=C_{2}^{w f}=C^{w f}$ for a symmetric system with $N=100$ signal space dimensions, transmitted power $P=10$, moderate interference gain $g=0.5$ and noise power $\eta_{0}=0.01$ for $k$ ranging from 1 to 99 dimensions $^{2}$ in FIGURE 3 and see that as the number of signal space dimensions $k$ occupied by transmitter 1 increases, $C_{1}^{s p}$ increases and $C_{2}^{s p}$ decreases - an obvious result. Less obvious, we see that for a wide range of values for $k$, both transmitters achieve higher capacities $C_{1}^{s p}$ and $C_{2}^{s p}$ if they partition the signal space than the capacity $C^{w f}$ corresponding to the simultaneous water filling solution. We also see that the collective capacity when transmitters partition the signal space $\mathcal{C}_{s p}$ does not vary widely with $k$. We thus form the impression that for moderate interference, both transmitters can often do much better under segregation than they would under simultaneous water filling, thus suggesting that the Nash equilibrium point implied by simultaneous water filling is an inefficient one.

Of course, the illustration begs the question of what constitutes moderate vs. weak interference. To this end we note that simultaneous water filling collective capacity $\mathcal{C}_{w f}^{*}$ depends on both the interference gain $g$ and the (raw) transmitter $\operatorname{SNR} \rho$, whereas signal space partitioning collective capacity $\mathcal{C}_{s p}$ depends only on $\rho$. We can plot these two capacity surfaces as a function of $g$ and $\rho$ as in FIGURE 4: the left hand side plot has a linear scale for $g$, while the right hand side plot has a logarithmic scale for $g$ and "magnifies" the interval $g<0.5$. Qualitatively, we note that only for small values of the interference gain $g$ does simultaneous water filling outperform segregation. Otherwise, the collective capacity under signal space partitioning is larger than for simultaneous water filling. This observation can be quantified by comparing the two capacity

\footnotetext{
${ }^{2}$ The values $k=1$, respectively $k=99$, correspond to the extreme cases in which transmitter 1 , respectively transmitter 2 , signal in only one signal dimension.
} 
expression in equation (45) and equation (49)

$$
\frac{N}{2} \log [1+2 \rho] \underset{\substack{\text { segregate } \\ \text { share }}}{\gtrless} N \log \left(1+\frac{\rho}{\rho g+1}\right)
$$

which simplifies to

$$
\rho \underset{\substack{\text { segregate } \\ \text { share }}}{\gtrless} \frac{1-2 g}{2 g^{2}}
$$

Since the raw $\operatorname{SNR} \rho$ is positive, equation (51) is always satisfied for $0.5 \leq g<1$, and one can define moderate interference as corresponding to interference gain values in the interval $[0.5,1)$, for which segregation outperforms simultaneous water filling, and is always preferable when $g>0.5$. In general the critical value of $g$ for a given $\operatorname{SNR} \rho$ is

$$
g^{*}=\frac{\sqrt{1+2 \rho}-1}{2 \rho}
$$

and for any $g>g^{*}$, segregation is preferable. For instance, for the numerical example considered in FIGURE 2, with $\rho=10$, we have $g^{*}=0.18$.

We close this section by noting that, assuming moderate interference and segregation, the necessary condition for maximizing the collective capacity in equation (47) is [25], [29]

$$
\frac{P_{1}}{k}=\frac{P_{2}}{N-k}
$$

and is similar to the result obtained in [4] using a different performance criterion than the collective capacity used in this work.

\section{B. Strong Interference}

As can be seen from FIGURE 2, "strong interference" $\left(g_{1} g_{2} \geq 1\right)$ implies multiple solutions for which capacities of both transmitters can vary widely. The most interior point of the simultaneous water filling region corresponds to complete spectral overlap in signal space, the points inside the region correspond to incomplete spectral overlap, and points on the outer border correspond to spectral separation in signal space. For $g=1$ the outer border of the water filling region becomes a single point, and as $g$ increases, the outer border expands and more points corresponding to spectral separation in signal space are possible. Furthermore, the point corresponding to complete 
spectral overlap moves closer to the origin as $g$ increases. The points inside the water filling region are suboptimal with respect to achievable capacity since the largest capacity is achieved by points on the outer border. The worst case scenario is complete spectral overlap.

However, the fact remains that under strong interference, points on the boundary of the simultaneous water filling region are stable under greedy assumptions. Furthermore, from a system perspective, the collective capacity varies only moderately as a function of the amount of signal space alloted to one transmitter over another as seen in FIGURE 3. So the main objective from a system control standpoint is to find algorithms or methods which nudge transmitters toward the simultaneous water filling boundary [25], [30].

\section{DISCUSSION}

Our results have interesting system control implications. For instance, under weak interference $\left(g_{1} g_{2} \ll 1\right.$ and moderate signal to noise ratio in a symmetric system), we have found that essentially ignoring interference and water filling over the entire signal space provides good performance for each transmitter and maximum collective capacity as well. Under strong interference $\left(g_{1} g_{2} \geq 1\right)$, socially optimal segregatory Nash equilibria exist alongside strongly suboptimal but equally stable water-filling Nash equilibria. While the suboptimal equilibria are undesirable, the stability of the optimal equilibria hold out hope that algorithms could be devised which reward transmitters who limit their spectral use and punish those who spread too widely. Under moderate interference, $\left(g_{1} g_{2}<1\right)$, the water filling Nash equilibria can be strongly suboptimal from a collective capacity standpoint and more socially optimal segregatory solutions are inherently unstable.

Since all these interference regimes seem probable in unlicensed wireless environments populated by current and emerging technologies, it seems prudent to ask what types of control mechanisms should be developed for efficient system operation.

In environments where interferers must repeatedly interact (as opposed to one-time interactions) there are a variety of distributed control ideas which can be brought to bear - strategies such as "tit for tat" or "generous tit for tat" [2], [23]. These ideas are currently being explored in the context of mutually interfering systems for both moderate and strong interference [29], [30].

Alternatively, one could envision a "spectrum server/advisor" [6], [31] to which systems might turn to help mediate spectrum use. The effective spectrum server would monitor local 
environments, determine the type and strength of interference, and advise systems on the most efficient use of spectral resources or at least on iterative strategies which led to improved performance.

This more global view of a spectrum advisor begs a comparison of collaborative versus noncollaborative approaches since one could imagine that in certain circumstances (such as strong interference) collaborative decoding could produce impressive capacity gains. That is, in a world increasingly populated by smart wideband radios, the best advice might be to cooperate [16], [19], [27], [28], [39] as opposed to finding ways to independently share the signal space. Likewise, there are also scenarios where the increased complexity of collaboration or explicitly coordinating spectrum use might not be worth meager best-case capacity gains.

To this end, consider the example in FIGURE 5 which corresponds to a symmetric system with two transmitters for weak, moderate, and strong interference. In both plots of FIGURE 5 we have shown the maximum sum capacity line of the Gaussian MIMO multiple access channel that corresponds to a collaborative scenario computed as in [17] for transmit covariance matrices that maximize sum capacity in the collaborative scenario [28].

For moderate and strong interference $(g>0.5)$, we note that there is a significant difference between collaborative capacities corresponding to maximum sum capacity in the collaborative scenario and the non-collaborative capacities in achieved on the outer border of the simultaneous water filling region. The reason for this disparity is obvious since increased $g$ implies more power capture by the "alien" receiver. In contrast, for weak interference the difference in performance is much less pronounced so that from a systems perspective, there is little to be gained by employing more sophisticated multi-system collaboration or interference channel coding methods - we would be better served by finding ways to help transmitter to avoid one another.

\section{SUMmARY AND CONCLUSIONS}

We have considered a simple non-collaborative wireless system with two transmitters and two receivers with flat communication channels, in which greedy performance optimization by individual transmitters leads to simultaneous water filling solutions. We have investigated properties of different solutions in relation to the interference gains between transmitters and receivers, and have identified their spectral structure: completely overlapped where each transmitter's spectrum is white, partially overlapped where each transmitter's spectrum is white in the overlap region and 
also white in regions where there is no overlap but not white overall, and completely separated where each transmitter spectrum is white in its respective subspace. In addition, we have defined the simultaneous water filling region as the set of pairs of achievable capacities for all possible simultaneous water filling points, for given transmitted powers, interference gains, and signal space dimensions, and the collective capacity as a global performance measure which allowed a global perspective on system performance. We have also provided a more detailed investigation of "weak", "moderate", and "strong" interference, focusing on when simultaneous water filling achieves optimal system performance and when it does not.

For weak interference, simultaneous water filling is indeed optimal since mutual interference levels are comparable to or below the noise floor. For moderate interference, water filling can be strongly suboptimal, and signal space partitioning offers much better performance. For strong interference, simultaneous water filling can result in socially optimal resource sharing. However, without external guidance toward the outer border of the simultaneous water filling region, there are many stable suboptimal "traps" in which the competing systems could be caught. These considerations led to the notion of a spectrum server/advisor which could help mutually interfering systems to better utilize shared spectrum.

To better understand design tradeoffs, non-collaboration was compared by means of numerical examples to complete collaboration where receivers pool information for joint decoding. For strong interference, both systems would be best served by finding ways to pool information and collaborate as opposed to pursuing various signal space separation strategies [25], [29], [30], or even sophisticated joint coding/decoding methods [16], [19] which would only increase capacity incrementally. In contrast, for moderate to weak interference, signal space partitioning affords good performance and the incremental gains to be had by collaboration are relatively small. So some means of keeping transmissions out of each others' way seems to be indicated. Such methods might be centralized as in a spectrum "advisor/server" [6], [31] or could be distributed as in tit for tat or other repeated game strategies [2], [7], [11], [14], [23], [29], [30].

\section{ACKNOWLEDGMENTS}

The authors wish to thank the Associate Editor, Dr. Kit K. Wong, and the three anonymous reviewers for their constructive comments on the paper. 


\section{REFERENCES}

[1] R. Ahlswede. The Capacity Region of a Channel with Two Senders and Two Receivers. Annals of Probability, 2(5):805814 , October 1974.

[2] R. Axelrod and W. D. Hamilton. The Evolution of Cooperation. Science, 211:1390-1396, 1981.

[3] M. Bengtsson. From Single Link MIMO to Multi-User MIMO. In Proceedings 2004 IEEE International Conference on Acoustics, Speech, and Signal Processing - ICASSP 2004, volume 4, pages IV.697-IV.700, Montreal, Canada, May 2004.

[4] P. P. Bergmans and T. M. Cover. Cooperative Broadcasting. IEEE Transactions on Information Theory, IT-20(3):317-324, May 1974.

[5] R. S. Blum. MIMO Capacity With Interference. IEEE Journal on Selected Areas in Communications, 21(5):793-801, June 2003. Special Issue on MIMO Systems and Applications.

[6] M. Buddhikot, P. Kolodzy, S. Miller, K. Ryan, and J. Evans. DIMSUMNet: New Directions in Wireless Networking Using Coordinated Dynamic Spectrum Access. In Proceedings $6^{\text {th }}$ IEEE International Symposium on a World of Wireless Mobile and Multimedia Networks - WoWMoM'05, pages 78-85, Taormina - Giardini Naxos, Italy, June 2005.

[7] C. F. Camerer, T.-H. Ho, and J.-K. Chong. Sophisticated Experience-Weighted Attraction Learning and Strategic Teaching in Repeated Games. Journal of Economic Theory, 104(1):137-188, May 2002.

[8] A. B. Carleial. A Case Where Interference Does Not Reduce Capacity. IEEE Transactions on Information Theory, 21(6):569-570, September 1975.

[9] A. B. Carleial. Interference Channels. IEEE Transactions on Information Theory, 24(1):60-70, January 1978.

[10] A. B. Carleial. Outer Bounds on the Capacity of Interference Channels. IEEE Transactions on Information Theory, 29(4):602-60, July 1983.

[11] N. Clemens and C. Rose. Intelligent Power Allocation Strategies in an Unlicensed Spectrum. In Proceedings First IEEE International Symposium on New Frontiers in Dynamic Spectrum Access Networks - DySPAN 2005, pages 37-42, Baltimore, MD, 2005.

[12] M. Costa. On the Gaussian Interference Channel. IEEE Transactions on Information Theory, 31(5):607-615, September 1985.

[13] T. M. Cover and J. A. Thomas. Elements of Information Theory. Wiley-Interscience, New York, NY, 1991.

[14] R. Etkin, A. Parehk, and D. Tse. Spectrum Sharing for Unlicensed Bands. In Proceedings First IEEE International Symposium on New Frontiers in Dynamic Spectrum Access Networks - DySPAN 2005, pages 251-258, Baltimore, MD, 2005.

[15] D. Fudenberg and J. Tirole. Game Theory. MIT Press, Cambridge, MA, 1991.

[16] A. Goldsmith, S. Jafar, and G. J. Foschini. Exploring Optimal Multicellular Multiple Antenna Systems. In Proceedings 2002 IEEE Vehicular Technology Conference - VTC'02 Fall, volume 1, pages 261-265, Vancouver, Canada, September 2002.

[17] A. Goldsmith, S. A. Jafar, and S. Viswanath. Capacity Limits of MIMO Channels. IEEE Journal on Selected Areas in Communications, 21(5):684-702, June 2003. Special Issue on MIMO Systems and Applications.

[18] T. S. Han and K. Kobayashi. A New Achievable Rate Region for the Interference Channel. IEEE Transactions on Information Theory, 27(1):49 - 60, January 1981.

[19] S. V. Hanly and P. A. Whiting. Information-Theoretic Capacity of Multi-Receiver Networks. Telecommunications Systems, 1(1):1-42, 1993.

[20] R. A. Horn and C. A. Johnson. Matrix Analysis. Cambridge University Press, Cambridge, United Kingdom, 1985. 
[21] H. J. Landau and H. O. Pollack. Prolate Spheroidal Wave Functions, Fourier Analysis and Uncertainty - III: The Dimension of the Space of Essentially Time- and Band-Limited Signals. The Bell System Technical Journal, 41(4):1295-1335, July 1962.

[22] R. B. Myerson. Game Theory - Analysis of Conflict. Harvard University Press, Cambridge, MA, 1991.

[23] M. A. Nowak and K. Sigmund. Tit For Tat in Heterogeneous Populations. Nature, 355:250-253, 1992.

[24] D. C. Popescu and C. Rose. Interference Avoidance Methods for Wireless Systems. Kluwer Academic Publishers, New York, NY, 2004.

[25] O. Popescu. Interference Avoidance for Wireless Systems with Multiple Receivers. PhD thesis, Rutgers University, Department of Electrical and Computer Engineering, 2004. Thesis Director: Prof. C. Rose. Available online at http://www.winlab.rutgers.edu/ otilia/thesis.pdf.

[26] O. Popescu, D. C. Popescu, and C. Rose. Interference Avoidance for Capacity Optimization in Mutually Interfering Wireless Systems. In Proceedings $62^{\text {nd }}$ IEEE Vehicular Technology Conference - VTC 2005 Fall, volume 1, pages 593-597, Dallas, TX, September 2005.

[27] O. Popescu and C. Rose. Greedy SINR Maximization in Collaborative Multi-Base Wireless Systems. EURASIP Journal on Wireless Communications and Networking, 2004(2):201-209, December 2004. Special Issue on Multiuser MIMO Networks.

[28] O. Popescu and C. Rose. Sum Capacity and TSC Bounds in Collaborative Multi-Base Wireless Systems. IEEE Transactions on Information Theory, 50(10):2433-2438, October 2004.

[29] O. Popescu, C. Rose, and D. C. Popescu. Signal Space Partitioning Versus Simultaneous Water Filling for Mutually Interfering Systems. In Proceedings 2004 IEEE Global Telecommunications Conference - GLOBECOM'04, volume 5, pages 3128-3132, Dallas, TX, December 2004.

[30] O. Popescu, C. Rose, and D. C. Popescu. Strong Interference and Spectrum Warfare. In Proceedings $38^{\text {th }}$ Conference on Information Sciences and Systems - CISS 2004, pages 83-88, Princeton, NJ, March 2004.

[31] C. Raman, R. D. Yates, and N. B. Mandayam. Scheduling Variable Rate Links via a Spectrum Server. In Proceedings First IEEE International Symposium on New Frontiers in Dynamic Spectrum Access Networks - DySPAN 2005, pages 110-118, Baltimore, MD, 2005.

[32] C. Rose. CDMA Codeword Optimization: Interference Avoidance and Convergence Via Class Warfare. IEEE Transactions on Information Theory, 47(6):2368-2382, September 2001.

[33] C. Rose, S. Ulukus, and R. Yates. Wireless Systems and Interference Avoidance. IEEE Transactions on Wireless Communications, 1(3):415-428, July 2002.

[34] H. Sato. Two-User Communication Channels. IEEE Transactions on Information Theory, 23(3):295-304, May 1977.

[35] H. Sato. On the Capacity Region of a Discrete Two-User Channel for Strong Interference. IEEE Transactions on Information Theory, 24(3):377-779, May 1978.

[36] H. Sato. The Capacity of the Gaussian Interference Channel Under Strong Interference. IEEE Transactions on Information Theory, 27(6):786-788, November 1981.

[37] C. E. Shannon. Two-way Communication Channels. In Proceedings $4^{\text {th }}$ Berkeley Symposium on Mathematics, Statistics, and Probabilities, pages 611-644. University of California Press, Berkeley, 1961.

[38] G. Strang. Linear Algebra and Its Applications. Harcourt Brace Jovanovich College Publishers, San Diego, CA, third edition, 1988 . 
[39] D. Tse and S. Hanly. Multi-access Fading Channels. Part I: Polymatroid Structure, Optimal Resource Allocation and Throughput Capacities. IEEE Transaction on Information Theory, 44(7):2796 - 2815, November 1998.

[40] S. Verdú. Multiuser Detection. Cambridge University Press, Cambridge, United Kingdom, 1998.

[41] W. Yu. Competition and Cooperation in Multiuser Communication Environments. PhD thesis, Stanford University, Department of Electrical Engineering, 2002. Principal Advisor: Prof. J. M. Cioffi. Available online at http://www.comm.toronto.edu/ weiyu/thesis_2.pdf.

[42] W. Yu, J. M. Cioffi, and G. Ginis. Distributed Multiuser Power Control for Digital Subscriber Lines. IEEE Journal on Selected Areas in Communications, 20(5):1105-1115, June 2002.

[43] W. Yu, W. Rhee, S. Boyd, and J. M. Cioffi. Iterative Water-Filling for Gaussian Vector Multiple-Access Channels. IEEE Transactions on Information Theory, 50(1):145-152, January 2004.

[44] F. C. Zagare. Game Theory. Concepts and Applications. Sage Publications, Beverly Hills, CA, 1984.

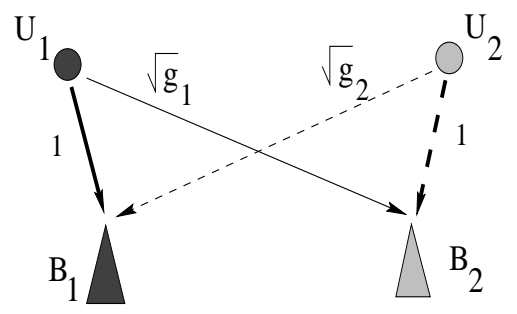

Fig. 1. A system with two transmitters and two receivers. $g_{1}$ is the interference gain corresponding to transmitter 1's signal at "alien" receiver 2 and $g_{2}$ is comparably defined. Gains to "home" receivers are normalized to 1.

TABLE I

\begin{tabular}{|c|c|c|c|}
\hline \multirow[b]{2}{*}{ Overlap } & \multicolumn{3}{|c|}{ Equilibrium Points } \\
\hline & 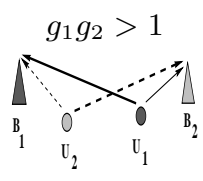 & $\underbrace{g_{1} g_{2}=1}_{\mathrm{V}_{1}}$ & 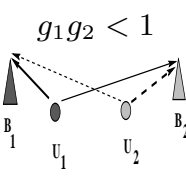 \\
\hline Complete & unique & unique $^{3}$ & unique \\
\hline Incomplete & many & many & - \\
\hline None & many & unique & - \\
\hline
\end{tabular}

${ }^{3}$ Except for the case $g_{1}=g_{2}=1$ when many equilibrium points are also possible as discussed in Section IV-A. 

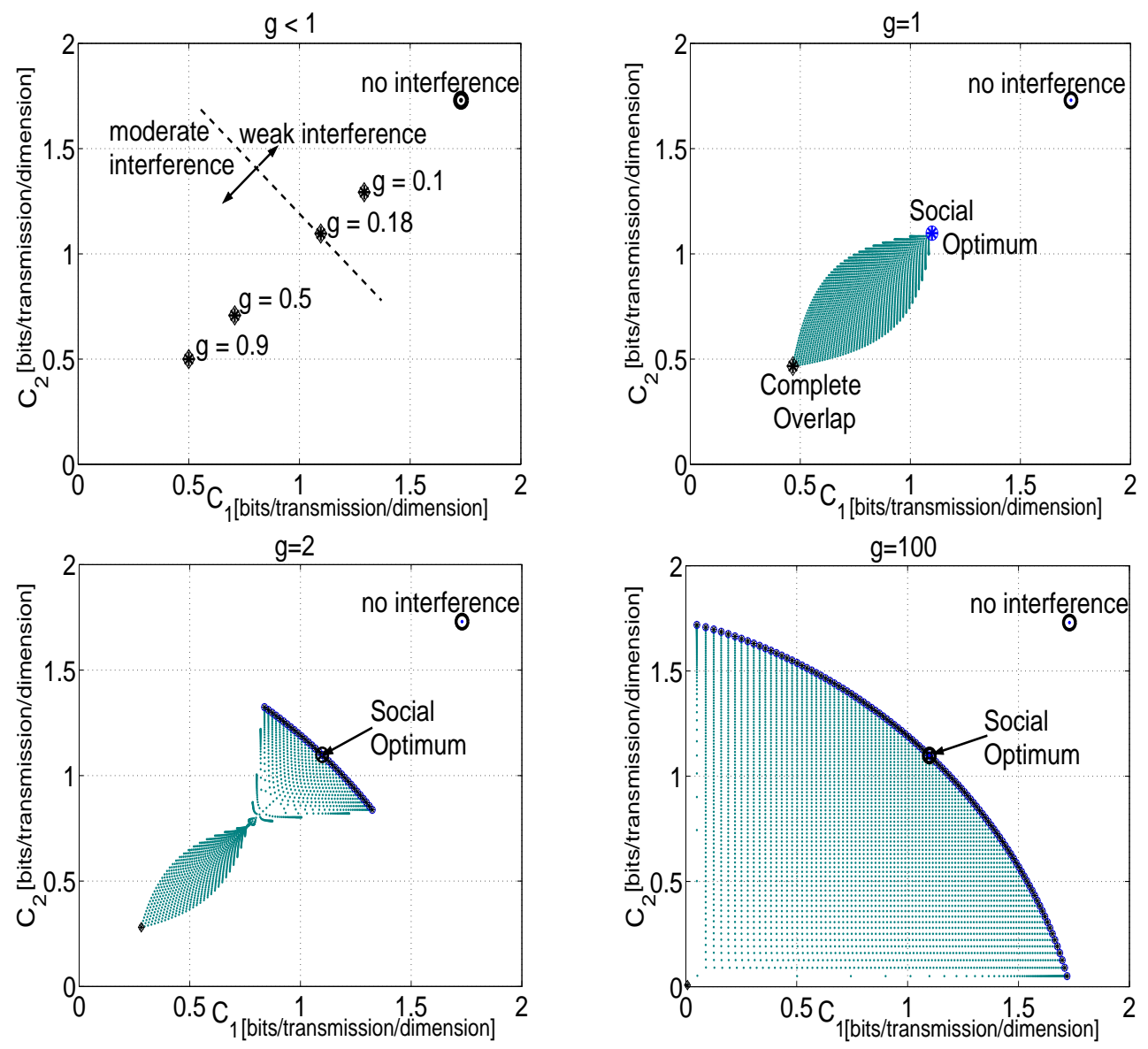

Fig. 2. Simultaneous Water Filling Region as a Function of Interference Gain. $N=100$ signal space dimensions, interference gains $g_{1}=g_{2}=g$, transmitted powers $P_{1}=P_{2}=10$, and noise floor $\eta_{0}=0.01$. 


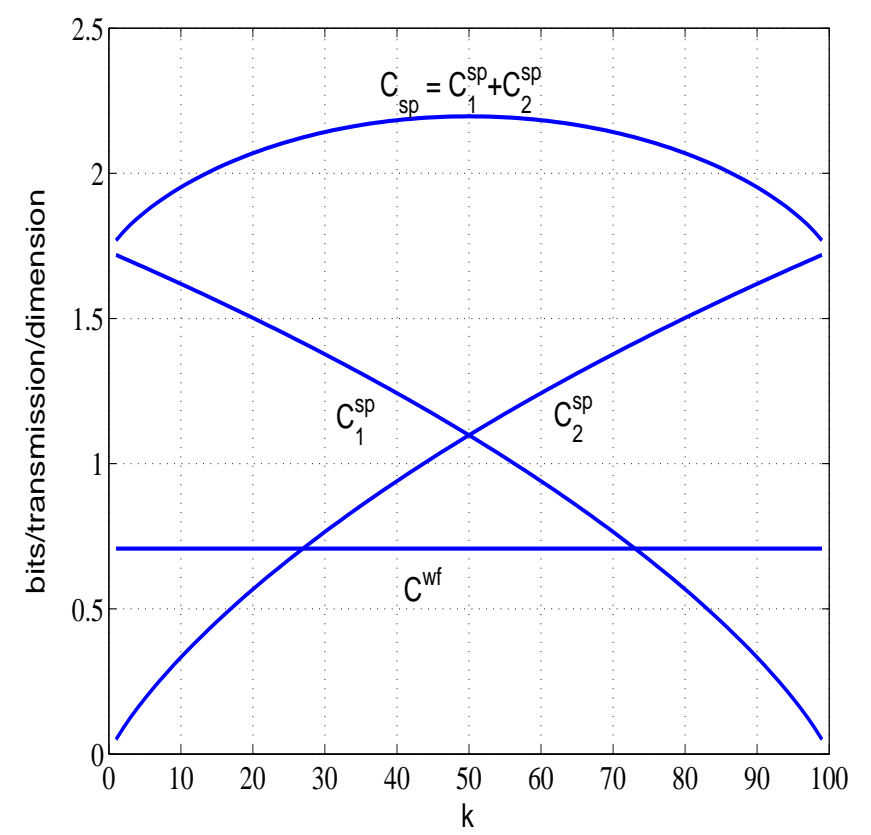

Fig. 3. Capacity variations as a function of signaling subspace width for moderate interference. $N=100$ signal space dimensions, transmitted powers $P_{1}=P_{2}=10$, interference gains $g_{1}=g_{2}=0.5$ and noise floor $\eta_{0}=0.01$.
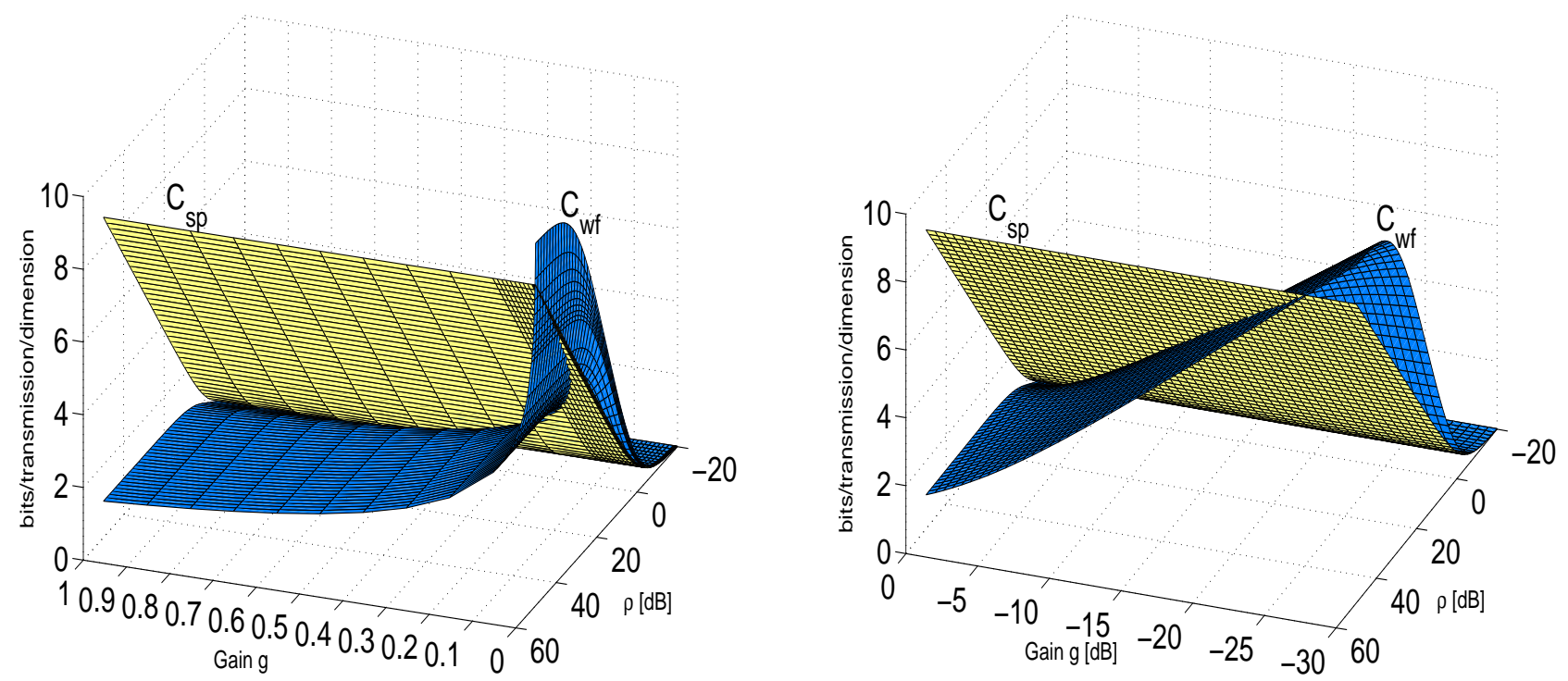

Fig. 4. Water filling and separation collective capacity as a function of interference gain and noise level for a symmetric system with $M=2$ transmitters. $N=100$ dimensions, user power $P=10$, interference gain ranges from $g=0.001$ to $g=1$, and background noise level $\eta_{0}$ ranges from $10^{-6}$ to 10 (so that $\rho$ ranges from $-20 \mathrm{~dB}$ to $50 \mathrm{~dB}$ ). 

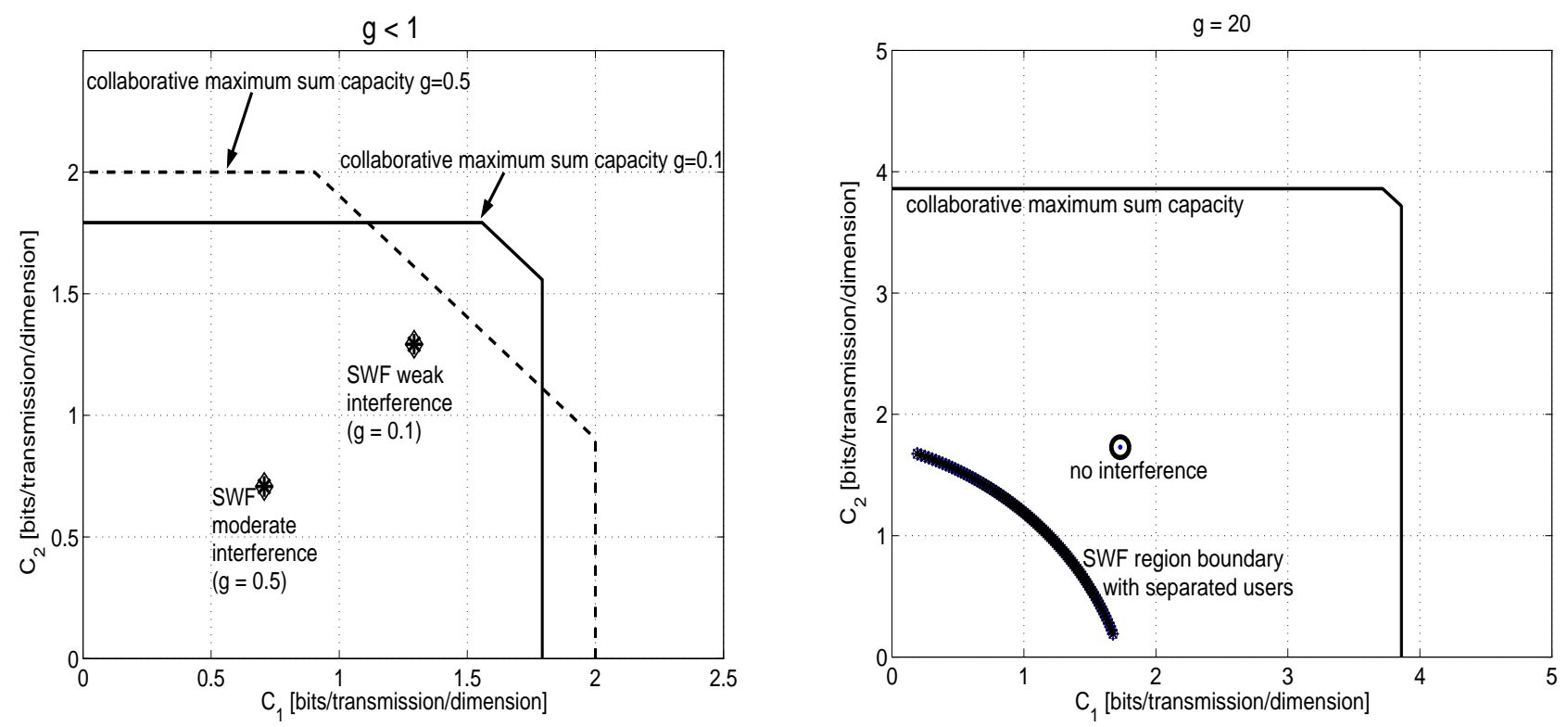

Fig. 5. A Comparison of Capacity Performance Between Collaborative Scenario and Non-Collaborative Simultaneous Water Filling (SWF) Scenario for Mutually Interfering Systems. Transmitted powers $P_{1}=P_{2}=P=10$, noise level $\eta_{0}=0.01$, and interference gains $g_{1}=g_{2}=0.1$ (weak interference), $g_{1}=g_{2}=0.5$ (moderate interference), and $g_{1}=g_{2}=20$ (strong interference). 